\title{
Proposta experimental do estudo de colisões entre bolas de borracha e superfície plana
}

Experimental proposal for the study of collision between rubber balls and flat surface

\author{
Francisco José Arnold*1, Alan Gabriel Godeny ${ }^{1}$, Murilo Matoso Costa ${ }^{1}$, João Francisco Viana ${ }^{1}$, \\ Rodrigo Luiz Ximenes ${ }^{1}$
}

${ }^{1}$ Universidade Estadual de Campinas, Faculdade de Tecnologia, Divisão de Telecomunicações, Limeira, SP, Brasil

Recebido em 02 de Maio, 2017. Revisado em 10 de Agosto, 2017. Aceito em 11 de Agosto, 2017.

\begin{abstract}
O estudo sobre a conservação do momento linear e as forças impulsivas nos cursos introdutórios de física tem abordagem baseada na colisão de objetos que se comportam como partículas. Neste trabalho busca-se por um aprofundamento no tema, introduzindo-se atividades experimentais direcionadas para laboratórios de ensino de física em que se considera a colisão entre uma bola de borracha e uma superfície rígida e plana. Estes experimentos permitem investigar características da colisão, tais como o tempo de impacto, a força impulsiva, a área de contato e o coeficiente de restituição. A modelagem, baseada na teoria de Hertz, inclui as propriedades elásticas da bola. Os resultados experimentais são comparados com valores teóricos provenientes do tratamento convencional (desconsiderando a deformação dos corpos) e da teoria de Hertz. Também são fornecidas indicações sobre a elaboração de experimentos visando aprimoramentos por parte de professores e alunos.
\end{abstract}

Palavras-chave: momento linear, força de impacto, teoria de Hertz.

The study on the conservation of linear momentum and impulsive forces in introductory physics courses has an approach based on the collision of objects that behave as particles. In this work we search for a deepening in the subject, introducing experimental activities directed to laboratories of physics teaching in which it is considered the collision between a rubber ball and a rigid and flat surface. These experiments allow us to investigate collision characteristics, such as the impact time, the impulsive force, the contact area and the coefficient of restitution. The modeling, based on the Hertz's theory, includes the elastic properties of the ball. The experimental results are compared with theoretical values from the conventional treatment (excluding the deformation of the bodies) and the Hertz theory. Indications are also given about the elaboration of experiments aiming at improvements by teachers and students.

Keywords: linear momentum, impact force, Hertz's theory.

\section{Introdução}

O estudo da colisão entre partículas é regido pelo princípio da conservação do momento linear e pelo Teorema do Impulso. A teoria acerca deste assunto é tratada em livros didáticos empregados em cursos de Física I [1-3]. Consideremos uma partícula, partindo do repouso e se movimentando em queda livre, isenta de forças dissipativas. Paulatinamente, ao longo da trajetória, ocorre uma conversão da energia potencial em cinética até que ocorra o choque com a superfície. Durante o choque atua uma força impulsiva que inverte o sentido do vetor velocidade e, por conseguinte, do momento linear da partícula. Posteriormente, ao iniciar um movimento ascendente, a energia cinética vai se convertendo em energia potencial e se anula quando a partícula alcança a posição com a mesma altura de onde foi solta. Essa situação, em que a energia mecânica é conservada, configura um choque perfeitamente elástico. Na prática, a partícula não retorna a

*Endereço de correspondência: arnold@ft.unicamp.br sua posição de origem. Isso significa que parte da energia é dissipada e, neste caso, o choque é inelástico. Ademais, o mesmo tratamento dado pelos livros didáticos à colisão entre partículas se estende para sistemas de partículas e corpos maciços. Neste caso, o centro de massa do objeto é assumido como uma partícula onde se concentra a propriedade da massa do mesmo.

A teoria da colisão entre corpos é um tema bastante antigo, no entanto, a descrição dos choques elásticos entre corpos deformáveis só foi desenvolvida por Hertz em 1881 e está apresentada no livro clássico de Love [4] de 1944. A teoria de Hertz se aplica apenas a choques perfeitamente elásticos e é considerada até hoje a base para a análise de diversos problemas ligados a colisões reportados na literatura especializada [5]. Esta teoria insere as propriedades elásticas dos corpos envolvidos no problema da colisão, diferenciando-se do tratamento costumeiramente apresentado nos livros didáticos [1-3], restrito a sistemas compostos por partículas que conservam suas distâncias de separação. 
Numa colisão, o coeficiente de restituição pode variar de 0 (choque totalmente inelástico) a 1 (choque perfeitamente elástico). Durante a colisão de uma bola com uma superfície perfeitamente rígida, pode-se dizer que a bola, devido a sua elasticidade, comporta-se de modo similar a uma mola: ela se deforma durante o impacto e retorna ao seu formato original após o mesmo. Logo após o início do impacto, a energia cinética da bola é gradativamente convertida em energia elástica e térmica durante a compressão até a velocidade do centro de massa ser nula. Em seguida, a energia elástica armazenada é retransformada em cinética e a bola passa a descrever uma trajetória ascendente. Em geral, em experimentos como este, as energias cinéticas antes e após o impacto são diferentes, de modo que é de se esperar que os resultados experimentais se ajustem razoavelmente bem à teoria de Hertz somente em situações em que o coeficiente de restituição esteja próximo de 1.

O estudo das colisões tem sido reportado na literatura sob diversos aspectos. Gugan [6], medindo a área e a duração do contato em colisões não elásticas de bolas com superfícies rígidas, constatou que, embora houvesse perda de energia cinética de cerca de $40 \%$, os resultados ainda assim eram compatíveis com a teoria desenvolvida por Hertz. Cross [7] analisou as diferenças nas colisões de bolas, molas e barras com superfícies planas. A colisão entre bolas visando a determinação de curvas de força e duração de impacto foi tratada em um artigo voltado ao desenvolvimento de instrumentação para o ensino de física [8]. Bacon et al. [9] estudaram colisões inelásticas de bolas em queda livre, mas restritas a alturas inferiores a $30 \mathrm{~cm}$.

Do ponto de vista do ensino, além de ser um tópico básico em física, estudar o movimento de queda livre de bolas reais é motivador e desperta atenção dos alunos devido à natural associação que se faz com o impacto de bolas usadas em atividades esportivas [10, 11]. Este tema, em particular, tem sido estudado por Cross [12] e pode ser abordado e adaptado em nível adequado de compreensão para alunos de cursos introdutórios de física básica.

A colisão unidimensional de uma bola em queda livre com um plano horizontal rígido é um experimento que pode ser facilmente executado em um laboratório de ensino de física. Em se tratando de objetos reais, tanto a bola como o plano sofrem deformações devido ao choque. Assim, a soma das energias cinética e potencial após o choque será menor do que antes, uma vez que parte delas são convertidas em energia interna. Tal constatação abre a possibilidade para o estudo da colisão entre esses corpos a partir de comparações entre as teorias com corpos rígidos e elasticamente deformáveis.

O objetivo principal do trabalho é apresentar experimentos, usando equipamentos de baixo custo, envolvendo colisões de bolas de borracha com uma superfície rígida, a partir da medida de parâmetros como o coeficiente de restituição, a duração do impacto, a altura da queda, a área de contato máxima durante o impacto e o raio da bola. Serão usados resultados provenientes de duas teorias: a primeira, que desconsidera as propriedades elásticas dos corpos envolvidos na colisão, tal como é feito nos cursos introdutórios de física, e a segunda, baseada na teoria de Hertz, onde os corpos apresentam propriedades elásticas.

Os conteúdos de cinemática e dinâmica presentes nas ementas da disciplina de Física I (em alguns casos até Física II) são bem conhecidas pelos professores. É sabido que a abordagem em relação a corpos deformáveis é restrita e, em alguns casos, principalmente em cursos de Engenharia, esse conteúdo é remetido à disciplina Resistência dos Materiais [13]. Dessa forma, este trabalho é direcionado a professores de cursos de física e afins que queiram reproduzir e aperfeiçoar experimentos sobre o entendimento do fenômeno das colisões usando-se duas abordagens teóricas, com e sem deformação dos corpos, e propondo atividades didáticas para treinamento em práticas de laboratório.

\section{Teoria}

A teoria sobre colisão abordada nos livros didáticos usa como premissa que os corpos envolvidos sejam tratados como partículas. Consideramos uma bola de massa $M$, lançada em queda livre, que colide perpendicularmente com uma superfície rígida, plana e com massa $M_{s}$ muito maior que $M$. Imediatamente antes do choque a bola possui velocidade $U$ e, durante o choque, sobre ela atua uma força contrária ao movimento que inverte o sentido de sua trajetória. A velocidade da bola após o choque, $U_{f}$, será igual a $U$, se o choque for perfeitamente elástico e será diferente de $U$, se o choque for imperfeitamente elástico.

A ação da força durante o contato tem uma duração $T$, bem curta, e é conhecida por força impulsiva. Se for levantado um gráfico da intensidade dessa força em função do tempo, a área da curva de $F_{e}$ no domínio do tempo define o impulso, $J$, em $(\mathrm{Ns})$, dado pela Equação (1)

$$
J=\int_{0}^{T} F_{e} d t
$$

Na maior parte dos casos, a função da força no tempo não é conhecida. Todavia, quando conhecemos a força média $\left(F_{m e d}\right)$ e a duração do contato, podemos determinar o módulo do impulso pela Equação (2).

$$
J=F_{m e d} T
$$

Os momentos lineares imediatamente antes e após o impacto são dados, respectivamente, por $P_{o}$ e $P_{f}$. Como o movimento da bola é unidimensional, o momento linear pode ser tratado como grandeza escalar considerando-se o sinal negativo para o movimento descendente e positivo para o ascendente. Sendo assim, o impulso é expresso em termos da variação do momento linear usando a Equação 
(3)

$$
J=\Delta P=P_{f}-\left(-P_{o}\right)
$$

Com isso, desprezando as forças dissipativas que surgem tanto na descida como na subida da bola e orientando o eixo y para cima, as velocidades associadas a esses momentos são dadas pela Equação de Torricelli (Equação $4)$.

$$
U_{f}^{2}=U^{2}-2 g \Delta y
$$

onde $g$ é a aceleração devido à gravidade e $U_{f}$ e $U$ são, respectivamente, as velocidades final e inicial da bola de cada etapa do movimento (descida e subida).

As Equações (1) a (3) expressam o conceito de impulso ligado à força de impacto e à conservação da quantidade de movimento e são estendidas para estudar problemas envolvendo sistemas de partículas e corpos maciços com suas propriedades físicas concentradas nos seus centros de massa. Por meio das Equações (2) e (3) determinamos a força média $\left(F_{m e d}\right)$ do impacto (Equação 5$)$.

$$
F_{\text {med }}=\frac{M\left(U_{f}-U\right)}{T}
$$

onde $M$ é a massa e $U_{f}$ e $U$ são as velocidades da bola de borracha imediatamente após e antes da colisão, respectivamente, calculadas pela Equação 4 .

Problemas envolvendo colisões de corpos que apresentam propriedades elásticas são estudados pela teoria de Hertz descrita em $[4,5,14,15]$. A teoria de Hertz estabelece como premissa que as deformações são pequenas e não ultrapassam o limite elástico dos corpos envolvidos e, com isso, toda a energia elástica armazenada pelos corpos durante a colisão é revertida posteriormente, excluindo-se do modelo qualquer tipo de forças dissipativas $[4,5]$. Na Figura 1 é mostrado um esboço de uma bola de raio $R$ colidindo com uma superfície perfeitamente rígida. No desenho, apresentado em dimensões exageradas, $r$ é o raio do círculo resultante no plano de contato devido à deformação na direção vertical $h$ (compliance).

Em [16] é descrita a teoria de Hertz para determinação da força de impacto de uma bola de massa $M$ e raio $R$, ao colidir, com velocidade $U$, em condições perfeitamente elásticas, com uma superfície plana. A força $\left(F_{e}\right.$ em N) e

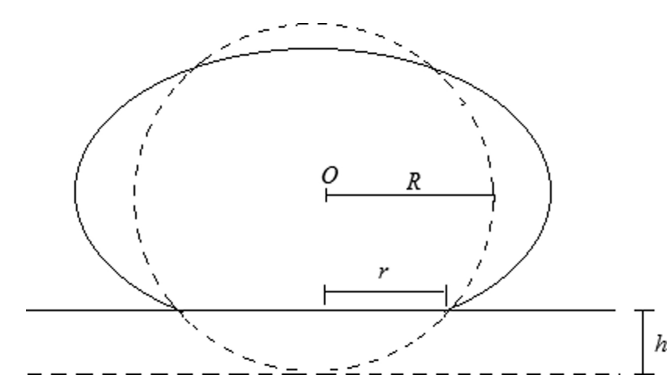

Figura 1: Esboço da colisão da bola com a superfície rígida. O desenho com linhas pontilhadas indica a situação sem colisão. O desenho com linhas cheias indica o momento de máxima deformação da bola na colisão. a área de contato $\left(A_{e} \mathrm{em} \mathrm{m}^{2}\right)$ são dados, respectivamente, pelas Equações (6) e (7) [16].

$$
\begin{gathered}
F_{e}=K R^{1 / 2} h^{3 / 2} \\
A_{e}=\pi R h
\end{gathered}
$$

onde

$$
K=\frac{4}{3}\left(\frac{1-\nu_{1}^{2}}{E_{1}}+\frac{1-\nu_{2}^{2}}{E_{2}}\right)^{-1}
$$

$K$ é dada em N $/ \mathrm{m}^{2}$.

$\nu_{1}$ e $\nu_{2}$ são os coeficientes de Poisson [17] da bola e da superfície de contato;

$E_{1}$ e $E_{2}$ são os módulos de Young da bola e da superfície de contato $\left(\mathrm{em} \mathrm{N} / \mathrm{m}^{2}\right)$.

A superfície de contato é considerada perfeitamente rígida, portanto, neste trabalho, $E_{2}$ tende ao infinito.

De acordo com [14], a força de impacto apresentada na Equação (9), possui a forma de uma meia senóide no intervalo de tempo entre $t=0$ e $t=T$ e é nula para tempos fora desse intervalo.

$$
F_{e}(t)=M h\left(\frac{\pi U}{2,94 h}\right)^{2} \operatorname{sen}\left(\frac{\pi U t}{2,94 h}\right)
$$

onde

$$
T=2,94 h / U
$$

$$
h=\left(\frac{5 M U^{2}}{4 R^{1 / 2} K}\right)^{2 / 5}
$$

A Equação (9) é uma boa aproximação em relação à correção sugerida em [15] que, converte a senóide em uma curva com forma similar a de um sino. Em termos de força média, a diferença dos resultados obtidos a partir das propostas apresentadas em [14] e [15] deve ser muito pequena.

Combinando-se as Equações 10 e 11 determinamos a constante $K$ definida pela Equação 8 e, com isso, podemos fazer uma estimativa do valor do módulo de Young da bola de borracha, desde que se conheça o coeficiente de Poisson. A Equação 12 exprime $K$ em função dos parâmetros de massa e raio da bola, duração do impacto e velocidade antes da colisão.

$$
K=\frac{18,52 M}{R^{1 / 2} U^{1 / 2} T^{5 / 2}}
$$

A força de impacto média é determinada pela Equação 13.

$$
\bar{F}_{e}=\frac{1}{T} \int_{0}^{T} F_{e}(t) d t
$$

Substituindo as Equações (9) e 10) em (13), obtemos a força média do impacto segundo a teoria de Hertz (Equação 14).

$$
F_{e}=\frac{2 \pi M}{2,94} \frac{U}{T}
$$


Manipulando algebricamente as Equações (8), (10) e (11), obtemos o deslocamento máximo do ponto tangente do início do contato da bola com a superfície rígida no sentido radial em $h$ e o tempo de duração do contato $T$ são dados pelas equações $(15)$ e $(16)$, respectivamente $[6]$

$$
\begin{gathered}
h^{5 / 2}=\frac{15 M U^{2} K}{16 R^{1 / 2}} \\
T=2,94\left(\frac{15 M K}{16 R^{1 / 2}}\right)^{2 / 5} U^{-1 / 5}
\end{gathered}
$$

A partir das Equações (7) e (15) obtemos uma relação entre a área de contato do impacto e a velocidade imediatamente anterior ao choque, como mostrado na Equação (17)

$$
A_{e}=a_{1} U^{4 / 5}
$$

onde $a_{1}$ é uma constante a ser determinada.

Pela Equação 16 verifica-se que

$$
T=b_{1} U^{-1 / 5}
$$

onde $b_{1}$ é uma constante a ser determinada.

As Equações 5 e 14 apresentam, portanto, duas formas de calcular a força média de impacto da bola de borracha com a superfície plana. Diferentemente da Equação 5, a força calculada a partir da teoria de Hertz (Equação 14) leva em conta as propriedades elásticas dos corpos.

$\mathrm{O}$ coeficiente de restituição, $e$, é vinculado à perda de energia cinética dos corpos em colisão. Este coeficiente depende das massas, das velocidades relativas e das formas dos corpos envolvidos [18]. O coeficiente de restituição aqui será restrito a considerar a dependência das velocidades relativas e é dado pela Equação 19

$$
e=\frac{U_{f}-V_{f}}{U-V}
$$

onde o numerador e o denominador da Equação 19 representam as velocidades relativas dos corpos (bola $\left(U \mathrm{e} U_{f}\right)$ e superfície $\left.\left(V \mathrm{e} V_{f}\right)\right)$ antes e após o choque. Neste trabalho, a superfície onde ocorre o choque é rígida e está imóvel, portanto, $V$ e $V_{f}$ são nulos.

Da mesma forma que se menciona a perda da energia cinética pode-se estabelecer analogia com a perda de energia potencial quando este choque se dá num movimento de queda livre conforme proposto neste trabalho. Neste caso, o coeficiente de restituição é, alternativamente, dado pela Equação 20

$$
e=\sqrt{\frac{y_{f}}{y_{i}}}
$$

onde $y_{i}$ é a altura inicial de onde a bola foi solta e $y_{f}$ é a altura máxima atingida pela bola após se chocar com o plano rígido.

Os estudantes dos semestre iniciais dos cursos da área de exatas não possuem embasamento para o entendimento da teoria de Hertz. No entanto, a abordagem da colisão em termos de uma premissa qualitativa da teoria de Hertz, juntamente com o enfoque convencional, permite ao professor criar situações didáticas que viabilizem atividades para um estudo comparativo.

\section{Materiais e métodos}

Experimentos para estudo de queda livre são bem conhecidos nas práticas de laboratório de Física Experimental I. Nesta seção estão descritos dois experimentos complementares que conduzem a obtenção de resultados experimentais os quais serão inseridos nas equações definidas pela Teoria de Hertz e na modelagem convencional. Ao professor interessado em realizar os experimentos aqui apresentados, cabe salientar que os custos são baixos, envolve materiais facilmente encontrados no mercado e o manuseio do experimento são acessíveis aos estudantes dos primeiros semestres dos cursos. Há também a possibilidade de criar adaptações e utilizar outras técnicas metodológicas que permitirão a determinação das grandezas necessárias para os cálculos de comparação das teorias.

Uma bola de borracha é lançada verticalmente, em queda livre, de uma determinada altura. Após a colisão com o solo, considerado perfeitamente rígido, ocorre uma restituição parcial de energia mecânica que eleva a bola verticalmente até um ponto de máxima altura. A altura do lançamento e a altura máxima alcançada após a colisão são alturas críticas com as quais é possível determinar as velocidades usadas na teoria (Equação 4) e o coeficiente de restituição (Equações 19 e 20). As medições, tanto das alturas, como das velocidades, podem ser realizadas usando-se técnicas ópticas $[6,19]$ ou imagens [20, 21]. O tempo necessário para inversão da velocidade (ou sentido de deslocamento) também é uma grandeza importante no estudo das colisões e pode ser medido a partir de experimentos com dispositivos piezelétricos $[11,12]$, imagens de alta velocidade $[11,20]$ ou contato elétrico [11, 22]. A deformação da bola durante o impacto (compliance) é determinada pela área de contato na forma de círculo que decorre da deformação da bola ao chocar-se com a superfície plana (Equação 7).

Nos experimentos foram utilizadas duas bolas de borracha maciças usadas como brinquedo para cães. A primeira bola, a ser tratada como SP1, possui diâmetro de 8,0 $\mathrm{cm}$, massa de $375,2 \mathrm{~g}$ e densidade de $1400,3 \mathrm{~kg} / \mathrm{m}^{3}$. A segunda bola, SP2, possui diâmetro de $5,6 \mathrm{~cm}$, massa de $133,9 \mathrm{~g}$ e densidade de $1456,2 \mathrm{~kg} / \mathrm{m}^{3}$. As medições de massa e de diâmetro apresentam incertezas de $\pm 0,1$ g e $\pm 0,1 \mathrm{~cm}$, respectivamente. A superfície plana onde ocorre a colisão é o piso do laboratório, revestido com granelite, que é considerado perfeitamente rígido.

Foram implementadas duas configurações experimentais para a realização das medições. Na primeira são medidas as alturas críticas e as áreas de contato. Na segunda são feitas as medições das durações do contato da bola com o solo. 


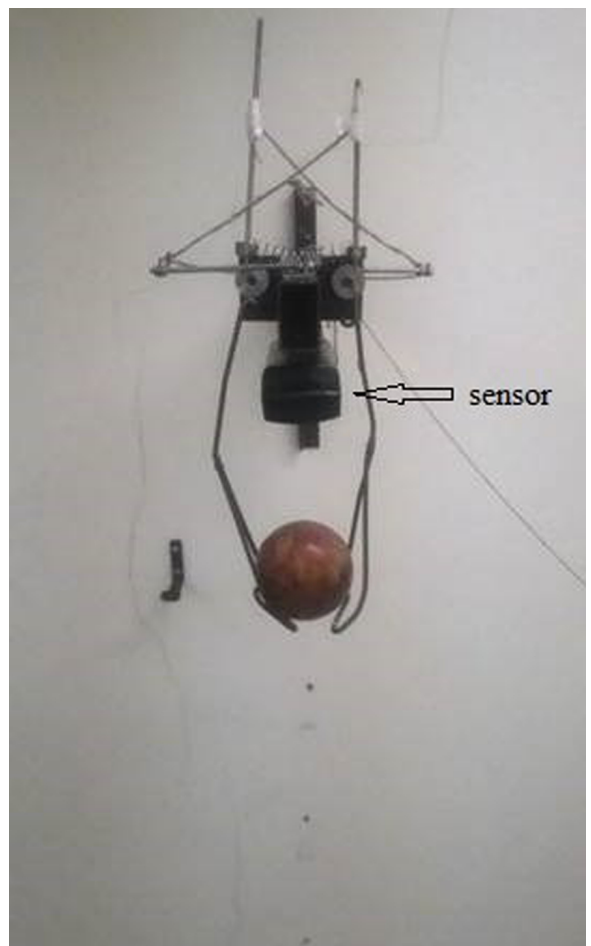

Figura 2: Dispositivo de lançamento da bola com sensor ultrassônico.

Inicialmente foi construído um dispositivo mecânico que pode ser posicionado em diferentes alturas e lança a bola a partir de um acionamento mecânico manual. Na Figura 2 é apresentada uma fotografia do sistema de lançamento. Cada bola foi lançada 12 vezes de 7 alturas diferentes. Um sensor ultrassônico da marca Go Motion, fabricado pela Vernier, foi usado para medir as distâncias em todo o percurso das bolas, desde seu lançamento até atingir a altura máxima após a colisão. Os dados colhidos pelo sensor são armazenados em um computador na forma de arquivo. A incerteza da medição feita pelo sensor é de $\pm 0,1 \mathrm{~cm}$. Os lançamentos foram feitos cuidadosamente, de modo a garantir que o movimento da bola tenha ocorrido, aproximadamente, em linha reta vertical e livre de efeitos indesejados de rotação e de impulsão inicial.

O princípio de operação do sensor ultrassônico baseiase na emissão e na recepção de ondas acústicas. No modo emissão, ele emite trens de pulsos sequenciais a cada $50 \mathrm{~ms}$ que viajam pelo ar até encontrar a bola de borracha, onde é refletido. Após a emissão de cada trem de pulsos, circuitos eletrônicos internos ao sensor promovem a conversão para o modo recepção, habilitandoo a receber as ondas refletidas. O processamento interno dos dados determina as posições da bola ao longo de toda trajetória. Do arquivo contendo o conjunto dessas posições são selecionadas as alturas críticas com as quais serão determinadas as velocidades imediatamente antes e após o impacto por meio da Equação 4. O valor da aceleração da gravidade usado é $g=9,7879 \mathrm{~m} / \mathrm{s}^{2}$, obtido a partir das equações apresentas em [23] da latitude local.

Antes de cada lançamento a bola foi pintada com tinta para carimbos. Essa pintura foi necessária para que, ao ocorrer o contato com o solo, fosse deixada uma marca circular correspondente à área de impacto em uma folha de papel. As marcas deixadas no papel apresentaram formas circulares e uniformes, cujo diâmetro foi medido com uma régua comum. Foram feitos testes preliminares que indicaram que a presença da folha de papel não altera a marca de tinta resultante do contato [22]. A incerteza da medição é de $\pm 0,5 \mathrm{~mm}$.

Como o tempo de impacto é da ordem de alguns milisegundos, o sensor ultrassônico não possui resolução suficiente para discriminar valores dessa magnitude. Esta foi a razão para a construção de um segundo experimento para medição do tempo de impacto.

O experimento para a medição do tempo de impacto da bola com o solo foi baseado na duração de um contato elétrico [22]. As bolas de borracha foram encapadas com uma fita adesiva de cobre. Nesta fita foi soldado um fio fino e flexível que se constitui como um terminal elétrico. Tanto a fita adesiva como o fio flexível possuem massas desprezíveis e não influem significativamente na trajetória da bola. A superfície de contato (solo) foi revestida com papel alumínio de espessura desprezível, formando-se um segundo terminal elétrico. Ao tocar o solo é fechada a malha de um circuito série constituído por uma fonte de alimentação de $5 \mathrm{~V}$, pela chave definida pelos terminais elétricos e por um resistor limitador de corrente de $1 \mathrm{k} \Omega$. Um osciloscópio Agilent DSO9254A, ajustado com uma varredura de $100 \mathrm{~ms}$ por divisão, foi conectado ao resistor e, ao registrar o evento elétrico do contato, permite que se meça o tempo em que bola e solo ficaram em contato.

Preliminarmente, com intuito de avaliar a condição de massa infinita do solo, o revestimento de cobre da bola foi pintado com tinta para carimbos e lançamentos foram realizados sobre o solo com e sem a folha de papel alumínio. Não foram detectadas diferenças nas áreas das marcas deixadas pela bola durante o impacto concluindose, portanto, que a condição de massa infinita do solo prevalece independentemente da presença da folha de alumínio.

Em seguida, para cada bola e cada altura foram realizados 12 lançamentos. As alturas de lançamento foram as mesmas das realizadas no experimento com o sensor ultrassônico. Os resultados foram obtidos a partir dos valores médios das medições. Um esboço esquemático desta montagem é mostrado na Figura 3.

A técnica ultrassônica usada para medir as alturas críticas não pôde ser usada no mesmo experimento para medição da duração do impacto. Isto se deve à reflexão indesejada causada pelo fio conectado a bola de borracha que impede a medição correta das alturas.

A metodologia de análise dos resultados se divide em quatro partes: 


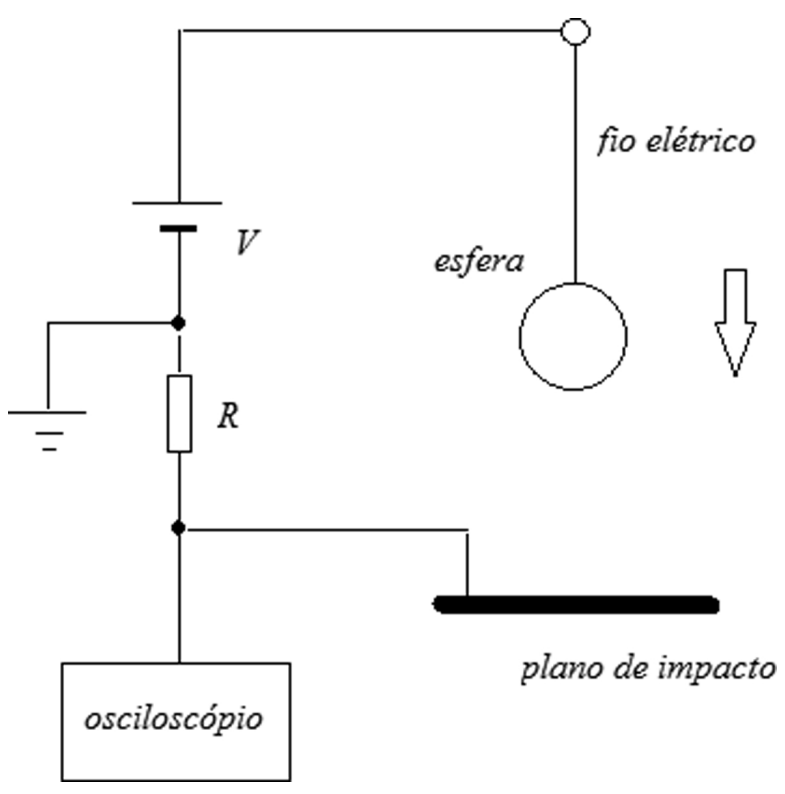

Figura 3: Diagrama de blocos do sistema montado para medir o tempo de impacto da bola com o plano horizontal.

\subsection{Determinação das forças de impacto}

A partir de resultados obtidos dos procedimentos descritos e da teoria apresentada é permitido determinar as forças de impacto médias e suas incertezas a partir das Equações 5 e 14. A propagação das incertezas ocorrida no cálculo das forças foi determinada pelas fórmulas apresentadas em [24]. Os resultados obtidos são comparados de modo que se possa avaliar não apenas a relação entre os valores, mas também as características das bolas de borracha que melhor se ajustem à teoria de Hertz.

\subsection{Determinação do módulo de Young das bolas de borracha}

$\mathrm{O}$ valor de $K$ é determinado com a Equação 12 para cada bola e cada altura de lançamento. A partir dos valores de $K$ é possível estimar o módulo de Young da borracha de cada bola (SP1 e SP2) com a Equação 8. $\mathrm{Na}$ Equação 8 se considera que o módulo de Young da superfície, $E_{2}$, é muito maior que o da bola de borracha, $E_{1}$, portanto a parcela de $K$ influenciada por $E_{2}$ é desprezível. O coeficiente de Poisson das borrachas em geral apresenta valores entre 0,45 e 0,50 [25]. Foram calculados valores de $E_{1}$ para todos os lançamentos e para os dois valores limites do coeficiente de Poisson. Desse conjunto de valores foi determinado o valor médio e o desvio padrão para cada bola. A realização desta metodologia conduz à caracterização do material constituinte da bola de borracha.

\subsection{Análise gráfica das curvas de ajuste do tempo e da área de contato com a velocidade de impacto}

As Equações 16 e 17 são usadas para obter curvas de ajuste que permitem uma avaliação sobre a validade da teoria de Hertz [6]. Os resultados experimentais da velocidade antes do impacto e a duração e a área de contato foram ajustados às funções usando-se o software Origin 8 (OriginLab Corporation). A convergência das curvas experimentais por meio dos coeficientes calculados dessas funções permite avaliar a teoria de Hertz em relação ao uso de bolas borracha comerciais nos experimentos realizados.

\subsection{Determinação do coeficiente de restituição}

A partir do cálculo do coeficiente de restituição (Equações 19 e 20) é possível avaliar a aproximação deste valor em relação a condição perfeitamente elástica do choque $(e=1)$. Portanto, neste item é verificado se o coeficiente de restituição se aproxima da situação elástica como necessário para atender a teoria de Hertz.

\section{Resultados e discussões}

Nas Tabelas 1 e 2 são apresentados os resultados experimentais das alturas, juntamente com as velocidades e coeficientes de restituição calculados, e os tempos de impacto de SP1 e SP2, respectivamente.

\subsection{Comparação das forças de impacto}

As Tabelas 3 e 4 apresentam as forças impulsivas, calculadas pelas teorias apresentadas, e a razão entre ambas, de SP1 e SP2, respectivamente.

Os valores obtidos para a duração do impacto estão na mesma ordem de valores relatados em [11] para medições

Tabela 1: Valores médios e desvios padrão das alturas de lançamento, das alturas atingidas após o contato com o solo ( $\mathrm{m}$ ), das velocidades de impacto $(U)(\mathrm{m} / \mathrm{s})$ e após o impacto $\left(U_{f}\right)(\mathrm{m} / \mathrm{s})$, dos coeficientes de restituição e dos tempos de duração do impacto (ms) de SP1.

\begin{tabular}{lllllll}
\hline exp. & $\Delta y(\mathrm{~cm})$ & $\Delta y_{r}(\mathrm{~cm})$ & $U(\mathrm{~cm} / \mathrm{s})$ & $U_{f}(\mathrm{~cm} / \mathrm{s})$ & $e$ & $T(\mathrm{~ms})$ \\
\hline 1 & $21,6 \pm 0,1$ & $19,1 \pm 1,9$ & $-205,6 \pm 0,5$ & $193,3 \pm 9,6$ & $(9,4 \pm 0,5) \times 10^{-1}$ & $5,6 \pm 0,2$ \\
\hline 2 & $52,2 \pm 0,1$ & $41,5 \pm 2,4$ & $-319,7 \pm 0,3$ & $285,0 \pm 8,2$ & $(8,9 \pm 0,3) \times 10^{-1}$ & $5,1 \pm 0,1$ \\
\hline 3 & $80,2 \pm 0,1$ & $61,3 \pm 0,9$ & $-396,2 \pm 0,2$ & $346,4 \pm 2,5$ & $(8,7 \pm 0,1) \times 10^{-1}$ & $4,9 \pm 0,1$ \\
\hline 4 & $105,5 \pm 0,1$ & $76,8 \pm 2,6$ & $-454,4 \pm 0,2$ & $387,7 \pm 6,6$ & $(8,5 \pm 0,1) \times 10^{-1}$ & $4,7 \pm 0,1$ \\
\hline 5 & $130,0 \pm 0,1$ & $93,0 \pm 1,4$ & $-504,4 \pm 0,2$ & $426,7 \pm 3,2$ & $(8,5 \pm 0,1) \times 10^{-1}$ & $4,6 \pm 0,1$ \\
\hline 6 & $156,7 \pm 0,1$ & $109,3 \pm 1,9$ & $-553,8 \pm 0,2$ & $462,6 \pm 4,0$ & $(8,3 \pm 0,1) \times 10^{-1}$ & $4,6 \pm 0,1$ \\
\hline 7 & $181,1 \pm 0,1$ & $123,4 \pm 2,4$ & $-595,4 \pm 0,2$ & $491,5 \pm 4,8$ & $(8,2 \pm 0,1) \times 10^{-1}$ & $4,5 \pm 0,1$ \\
\hline
\end{tabular}


Tabela 2: Valores médios e desvios padrão das alturas de lançamento, das alturas atingidas após o contato com o solo ( $\mathrm{m}$ ), das velocidades de impacto $(U)(\mathrm{m} / \mathrm{s})$ e após o impacto $\left(U_{f}\right)(\mathrm{m} / \mathrm{s})$, dos coeficientes de restituição e dos tempos de duração do impacto (ms) de SP2.

\begin{tabular}{lllllll}
\hline exp. & $\Delta y(\mathrm{~cm})$ & $\Delta y_{r}(\mathrm{~cm})$ & $U(\mathrm{~cm} / \mathrm{s})$ & $U_{f}(\mathrm{~cm} / \mathrm{s})$ & $e$ & $T(\mathrm{~ms})$ \\
\hline 1 & $24,5 \pm 0,1$ & $21,3 \pm 1,4$ & $-219,0 \pm 0,4$ & $204,2 \pm 6,7$ & $(9,3 \pm 0,3) \times 10^{-1}$ & $3,9 \pm 0,1$ \\
\hline 2 & $54,3 \pm 0,1$ & $44,4 \pm 2,1$ & $-326,0 \pm 0,3$ & $294,8 \pm 7,0$ & $(9,0 \pm 0,2) \times 10^{-1}$ & $3,5 \pm 0,1$ \\
\hline 3 & $82,6 \pm 0,1$ & $62,8 \pm 1,0$ & $-402,1 \pm 0,2$ & $350,6 \pm 2,8$ & $(8,7 \pm 0,1) \times 10^{-1}$ & $3,4 \pm 0,2$ \\
\hline 4 & $107,9 \pm 0,1$ & $79,6 \pm 1,9$ & $-460,0 \pm 0,2$ & $394,7 \pm 4,7$ & $(8,6 \pm 0,1) \times 10^{-1}$ & $3,1 \pm 0,1$ \\
\hline 5 & $132,4 \pm 0,1$ & $97,5 \pm 1,7$ & $-509,1 \pm 0,2$ & $436,9 \pm 3,8$ & $(8,6 \pm 0,1) \times 10^{-1}$ & $2,9 \pm 0,2$ \\
\hline 6 & $159,2 \pm 0,1$ & $111,4 \pm 2,6$ & $-558,2 \pm 0,2$ & $467,0 \pm 5,4$ & $(8,4 \pm 0,1) \times 10^{-1}$ & $2,9 \pm 0,3$ \\
\hline 7 & $183,5 \pm 0,1$ & $126,6 \pm 3,8$ & $-599,3 \pm 0,2$ & $497,8 \pm 7,5$ & $(8,3 \pm 0,1) \times 10^{-1}$ & $2,9 \pm 0,1$ \\
\hline
\end{tabular}

Tabela 3: Valores médios e desvios padrão da força impulsiva média da bola como corpo maciço $(\mathrm{N})$, da força impulsiva média da bola como corpo elástico $(\mathrm{N})$ e a razão dessas forças de SP1.

\begin{tabular}{llll}
\hline exp. & $\left|F_{\text {med }}\right|(\mathrm{N})$ & $\left|F_{e}\right|(\mathrm{N})$ & $F_{e} / F_{\text {med }}$ \\
\hline 1 & $(2,67 \pm 0,06) \times 10^{2}$ & $(2,94 \pm 0,01) \times 10^{2}$ & $1,10 \pm 0,03$ \\
\hline 2 & $(4,42 \pm 0,08) \times 10^{2}$ & $(4,99 \pm 0,07) \times 10^{2}$ & $1,13 \pm 0,03$ \\
\hline 3 & $(5,70 \pm 0,17) \times 10^{2}$ & $(6,49 \pm 0,20) \times 10^{2}$ & $1,14 \pm 0,05$ \\
\hline 4 & $(6,71 \pm 0,19) \times 10^{2}$ & $(7,73 \pm 0,21) \times 10^{2}$ & $1,15 \pm 0,04$ \\
\hline 5 & $(7,59 \pm 0,12) \times 10^{2}$ & $(8,79 \pm 0,13) \times 10^{2}$ & $1,16 \pm 0,02$ \\
\hline 6 & $(8,34 \pm 0,11) \times 10^{2}$ & $(9,71 \pm 0,13) \times 10^{2}$ & $1,16 \pm 0,02$ \\
\hline 7 & $(8,98 \pm 0,14) \times 10^{2}$ & $(10,51 \pm 0,16) \times 10^{2}$ & $1,17 \pm 0,03$ \\
\hline
\end{tabular}

Tabela 4: Valores médios e desvios padrão da força impulsiva média da bola como corpo maciço $(\mathrm{N})$, da força impulsiva média da bola como corpo elástico (N) e a razão dessas forças de SP2.

\begin{tabular}{llll}
\hline exp. & $\left|F_{\text {med }}\right|(\mathrm{N})$ & $\left|F_{e}\right|(\mathrm{N})$ & $F_{e} / F_{\text {med }}$ \\
\hline 1 & $(1,43 \pm 0,03) \times 10^{2}$ & $(1,59 \pm 0,03) \times 10^{2}$ & $1,10 \pm 0,03$ \\
\hline 2 & $(2,37 \pm 0,07) \times 10^{2}$ & $(2,66 \pm 0,08) \times 10^{2}$ & $1,12 \pm 0,05$ \\
\hline 3 & $(2,98 \pm 0,19) \times 10^{2}$ & $(3,40 \pm 0,22) \times 10^{2}$ & $1,14 \pm 0,10$ \\
\hline 4 & $(3,67 \pm 0,13) \times 10^{2}$ & $(4,21 \pm 0,15) \times 10^{2}$ & $1,15 \pm 0,06$ \\
\hline 5 & $(4,29 \pm 0,25) \times 10^{2}$ & $(4,94 \pm 0,28) \times 10^{2}$ & $1,15 \pm 0,09$ \\
\hline 6 & $(4,70 \pm 0,43) \times 10^{2}$ & $(5,47 \pm 0,51) \times 10^{2}$ & $1,16 \pm 0,15$ \\
\hline 7 & $(5,05 \pm 0,19) \times 10^{2}$ & $(5,89 \pm 0,22) \times 10^{2}$ & $1,17 \pm 0,06$ \\
\hline
\end{tabular}

com bolas macias usadas em atividades esportivas. Notamos também que a razão das forças de impacto calculadas com a teoria de Hertz estão, aproximadamente, entre $10 \%$ e $20 \%$ acima dos valores calculados pela teoria para corpos maciços. Essa faixa percentual obtida é um indicativo que as colisões com as bolas de borracha experimentadas estão muito próximas à condição de choque perfeitamente elástico. Portanto, a teoria de Hertz para esses casos produz resultados próximos aos obtidos por uma metodologia convencional com corpos maciços.

Verificamos também uma tendência de aumento da razão das forças com a velocidade de impacto. Portanto, nos impactos de maior intensidade a aproximação da condição de choque perfeitamente elástico tende a diminuir.

\subsection{Módulo de Young das bolas de borracha}

A média de valores para $E_{1}$ de SP1 e SP2 são de $(6,08$ $\pm 0,12) \mathrm{MPa}$ e $(7,14 \pm 0,83) \mathrm{MPa}$, respectivamente. Estes valores são compatíveis com a ordem de valores encontrados para o módulo de Young de borrachas [26]. Estes resultados indicam que a metodologia proposta também permite realizar uma estimativa sobre as propriedades elásticas dos materiais constituintes das bolas usadas nos lançamentos.

\subsection{Análise gráfica}

Na Figura 4 são mostradas as curvas do tempo de impacto em função da velocidade imediatamente antes da colisão de SP1 e SP2. As curvas foram ajustadas a partir da função $T=a|U|^{b}$. Foram obtidos os seguintes valores: $a=(6,5 \pm 0,1) \mathrm{ms}$ e $b=(-2,1 \pm 0,1) \times 10^{-1}$, para SP1 e $a=(5,1 \pm 0,2) \mathrm{ms}$ e $b=(-3,2 \pm 0,2) \times 10^{-1}$, para SP2.

$\mathrm{O}$ ajuste das curvas dos resultados experimentais mostram que a diferença dos valores de $b$ em relação ao previsto pela Equação 18 é menor para SP1 (bola de maior massa). A explicação desta melhor aderência à teoria de Hertz pode ser atribuída a um maior controle do experimento quando se utiliza bolas com maior raio (maior massa), como consequência da minimização dos efeitos da fiação e da capa metálica.

Na Figura 5 são mostradas as curvas da área de impacto em função da velocidade imediatamente antes da colisão de SP1 e SP2. As curvas foram ajustadas a partir

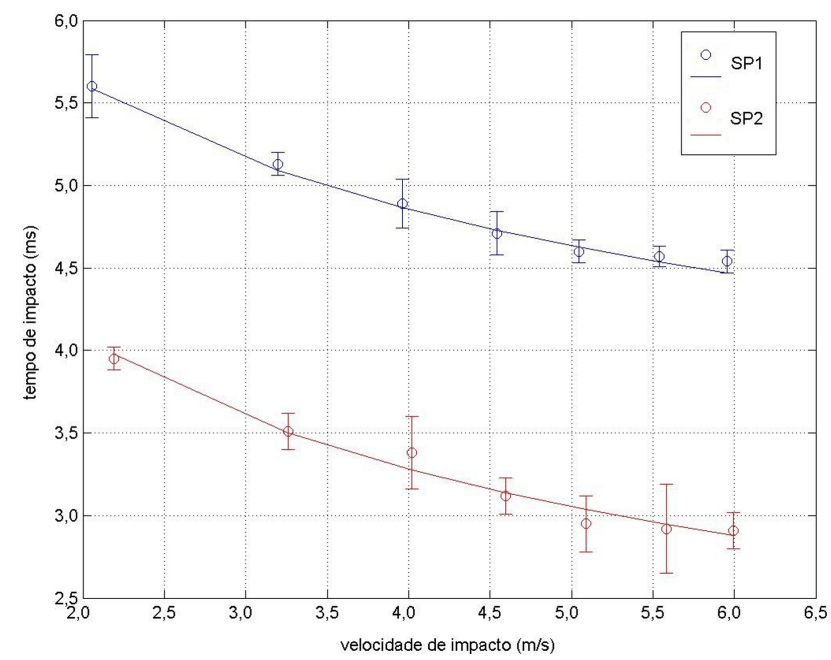

Figura 4: Tempo de impacto em função da velocidade de impacto. A incerteza da velocidade é menor que o tamanho do marcador. 


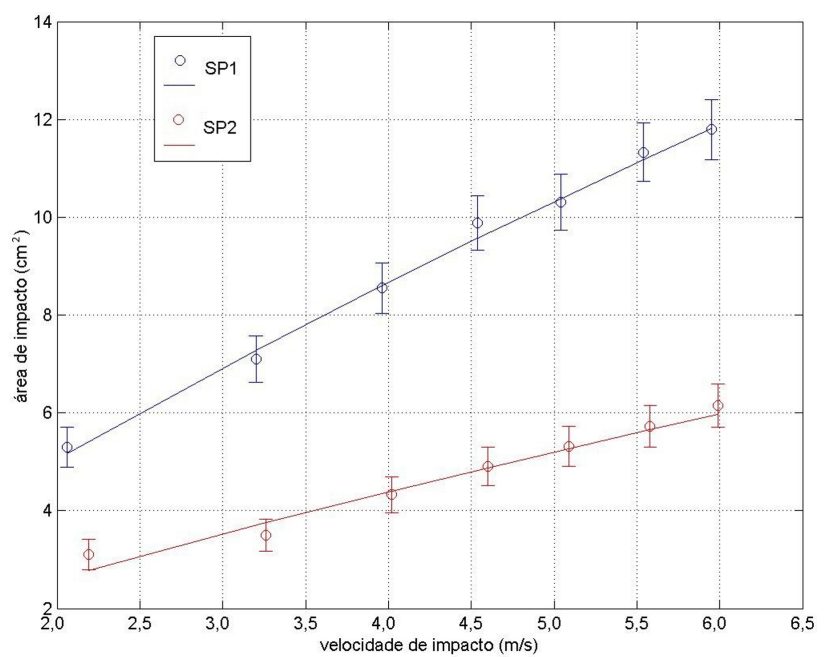

Figura 5: Área de impacto em função da velocidade de impacto para SP1 (traço azul) e SP2 (traço vermelho). A incerteza da velocidade é menor que o tamanho do marcador.

da função $A=c|U|^{d}$. Foram obtidos os seguintes valores: $c=(2,9 \pm 0,1) \mathrm{cm}^{2}$ e $d=(7,8 \pm 0,3) \times 10^{-1}$, para SP1 e $c=1,5 \pm 0,2) \mathrm{cm}^{2}$ e $d=(7,6 \pm 1,5) \times 10^{-1}$, para SP2.

Esses resultados mostram que, tanto para SP1, como para SP2, o expoente da Equação 17 se aproxima do previsto pela teoria de Hertz, ou seja, 0,8. Diferentemente do experimento que gerou as curvas da Figura 4, neste caso não houve a inclusão de conexões elétricas que pudessem afetar os resultados, especialmente em relação a SP2.

Os resultados experimentais apresentados nesta subseção mostram que o tempo de contato durante a colisão diminui com o aumento da altura do lançamento da bola (ou da velocidade de impacto). A área de contato da bola com a superfície plana aumenta com a velocidade de impacto. Ambos resultados se mostram coerentes com os obtidos em [6]. A duração e a área de contato são maiores quanto maior for o diâmetro da bola.

\subsection{Coeficiente de restituição}

A Figura 6 mostra as curvas do coeficiente de restituição em função da velocidade de impacto para as bolas SP1 e SP2.

Os valores obtidos para o coeficiente de restituição estão próximos aos determinados por [10] para bolas de tênis, futebol, basquete e golfe indicando, portanto, a similaridade de comportamentos das bolas empregadas.

De acordo com Sondergaard et al. [27], a partir dos resultados obtidos com lançamentos de bolas de aço com diâmetros de até $2,22 \mathrm{~cm}$, há uma distância crítica entre a altura do lançamento da bola e a placa rígida a partir da qual o coeficiente de restituição permanece constante. Em distâncias inferiores à crítica, o coeficiente de restituição é maior. Essa distância crítica aumenta linearmente em relação ao diâmetro da bola. Os resultados apresentados na Figura 6 não permitem concluir que os coeficientes

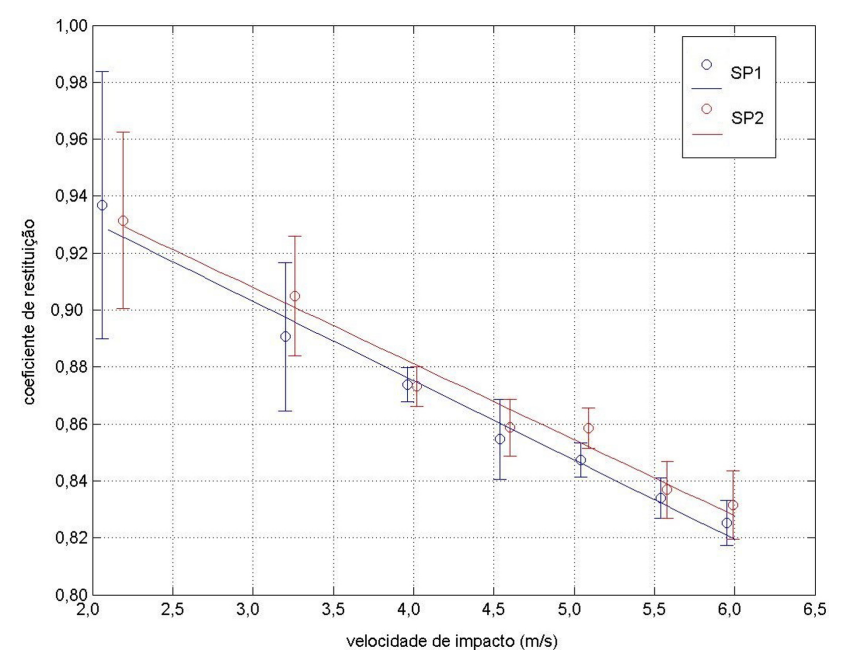

Figura 6: Coeficiente de restituição em função de velocidade de impacto. Traço azul: bola SP1. Traço vermelho: bola SP2. A incerteza da velocidade é menor que o tamanho do marcador.

de restituição de SP1 (bola com diâmetro maior) são inferiores aos de SP2 (bola com diâmetro menor), pois os intervalos de confiança dos resultados encontram-se sobrepostos. Também foi verificado que o coeficiente de restituição diminui com a velocidade de impacto e com a área de contato, pois estas são proporcionais (Equação 17). Não foi encontrado o valor limite da distância de lançamento como indicado em [27], mas em toda faixa de alturas de lançamento foi notada uma diminuição aproximadamente linear do coeficiente de restituição. Como em nossos experimentos foram usadas bolas de borracha com diâmetros superiores aos das bolas de aço usadas em [27], é possível que a distância crítica possa ser encontrada para lançamentos de alturas maiores.

Há coerência entre a diminuição do coeficiente de restituição e o aumento da taxa das forças calculadas com as teorias empregadas observadas nas Tabelas 3 e 4, pois são indicativos que o aumento da velocidade de impacto conduz a um afastamento das condições que caracterizam um choque perfeitamente elástico.

\section{Conclusão}

Foi apresentado um conjunto de experimentos, simples e de baixo custo, em que se permite estudar as colisões entre uma bola de borracha e um plano rígido levandose em conta as características elásticas da bola. Neste trabalho foram apresentados resultados que ilustram a adequação da teoria de Hertz em casos em que os coeficientes de restituição são superiores a $82 \%$, portanto, muito próximos aos casos perfeitamente elásticos.

Os experimentos apresentados são perfeitamente compreensíveis a alunos de semestres iniciais de cursos de física, conduzem a um entendimento, via teoria de Hertz, mais aprofundado e realista das colisões pela inserção das propriedades elásticas de um dos corpos e estimu- 
lam possíveis aperfeiçoamentos que professores podem realizar durante a prática diária do ensino.

A utilização de um sensor ultrassônico para medir as alturas críticas constitui uma inovação em relação a outras metodologias apresentadas na literatura especializada para esta finalidade. Professores e alunos interessados poderão adaptar e aperfeiçoar os experimentos de acordo com os materiais disponíveis em seus laboratórios.

Os resultados apresentados em IV.3 podem ser usados também como atividade de interpretação de dados em laboratório. Essa atividade contribui para o entendimento dos procedimentos de ajuste de curvas não lineares, uma vez que as funções de tempo e de área de impacto em função da velocidade apresentam essa característica (Eqs. (17) e (18)).

O comportamento de uma bola de borracha é satisfatoriamente aderente a teoria proposta por Hertz e o módulo de Young da mesma pode ser estimado neste tipo de problema. Conforme as discussões sobre os resultados do ajuste de curvas feito na subseção IV.3, é recomendado, no sentido de obter resultados próximos ao de um choque elástico e coerentes com a teoria de Hertz, usar bolas de borracha com massas e diâmetros mais elevados.

\section{Referências}

[1] D. Halliday, R. Resnick e J. Walker, Fundamentos de Física, vol. 1 (LTC, Rio de Janeiro, 2012), 9a ed.

[2] P.A. Tipler e G. Mosca, Física para Cientistas e Engenheiros, vol. 1 (LTC, Rio de Janeiro, 2000), $4^{\mathrm{a}}$ ed.

[3] P.R. Kesten e D.L. Tauck, Física na Universidade para as Ciências Físicas e da Vida, vol. 1 (LTC, Rio de Janeiro, 2015), $1^{\mathrm{a}}$ ed.

[4] A.E.H. Love, A Treatise on the Mathematical Theory of Elasticity (Dover Publications, New York, 1944), 4th ed.

[5] W. Goldsmith, Impact - The Theory and Physical Behaviour of Collinding Solids (Dover Publications, New York, 2001).

[6] D. Gugan, Am. J. of Phys. 68, 920 (2000).

[7] R. Cross, Am. J. Phys. 76, 908 (2008).

[8] R. Hessel, A.C. Perinotto, R.A.M. Alfaro and A.A. Freschi, Am. J. Phys. 74, 176 (2006).

[9] M.E. Bacon, B. Stevenson and C.G. Stafford Baines, Am. J. Phys. 66, 445 (1998).

[10] K.C. Maynes, M.G. Compton and B. Baker, The Phys. Teacher 43, 352 (2005).

[11] J.R. Roberts, R. Jones and S.J. Rothberg, Sports Engineering 4, 191 (2001).

[12] R. Cross, Am. J. Phys. 67, 222 (1999).

[13] T.C. Ramos E L. Vertchenko, Rev. Bras. Ens. Fis. 33, $1502(2011)$

[14] S.C. Hunter, J. Mech. Phys. Sol. 5, 162 (1957).

[15] J. Reed, J. Phys. D: Appl. Phys. 18, 2329 (1985).

[16] L. Kogut and I. Etsion, J. Appl. Mech. 69, 657 (2002).

[17] L.E. Kinsler, A.R. Frey, A.B. Coopers and J.V. Sanders, Fundamentals of Acoustics (John Wiley \& Sons, New York, 2000), 4th ed.

[18] W. Goldsmith, Bull. Mech. Div. Am. Soc. Eng. Educ. 2, 10 (1952).
[19] V.R. Coluci, G. Paulino, D.C. de Souza e E.P.R. Vasconcelos, Rev. Bras. Ens. Fis. 35, 2506 (2013).

[20] E.P.M. Corveloni, E.S. Gomes, A.R. Sampaio, A.F. Mendes, V.L.L. Costa e R.C. Viscovini, Rev. Bras. Ens. Fis. 31, 3504 (2009).

[21] C. Sirisathitkul, P. Glawtanong, T. Eadkong e Y. Sirisathitkul, Rev. Bras. Ens. Fis. 35, 1504 (2013).

[22] P. Muller, R. Böttcher, A. Russel, M. Trüe, S. Aman and J. Tomas, Adv. Pow. Tech. 27, 1233 (2016).

[23] W. Lopes, Cad. Bras. de Ens. de Fís. 25, 561 (2008).

[24] J.H. Vuolo, Fundamentos da Teoria de Erros (E. Blucher, São Paulo, 2015), $2^{\text {a }}$ ed.

[25] M. Caetano, Ciência e Tecnologia da Borracha, http://ctborracha.com/?page_id=1596, acessado em $7 / 6 / 2017$.

[26] M. Caetano, Ciência e Tecnologia da Borracha, http://ctborracha.com/?page_id=1586, acessado em $7 / 6 / 2017$.

[27] R. Sondergaard, K. Chaney and C.E. Brennen, J. App. Mech. 57, 694 (1990). 